\title{
MODIFIED KRATZER-FUES FORMULA FOR ROTATION-VIBRATION ENERGY OF DIATOMIC MOLECULES*
}

\author{
M. Molski AND J. KonarSKI \\ Department of Theoretical Chemistry, Faculty of Chemistry \\ A. Mickiewicz University \\ Grunwaldzka 6, 60-780 Poznań, Poland
}

(Received May 7, 1992; revised version September 1, 1992)

\begin{abstract}
An extension of the Kratzer-Fues approach to analytical calculation of the rotation-vibration energy of diatomic molecules is proposed. The eigenvalues from this approach are applied in calculation of the rotational and rovibrational energies and in evaluation of molecular constants of selected diatomic molecules, resulting in satisfactory reproduction of experimental frequencies over a wide range of rotational states. In contrast to our previous proposition the rotational dependence of vibration energy is taken into account. An additional set of fitted parameters which include equilibrium distance and dissociation constant was also employed.
\end{abstract}

PACS numbers: 33.10.Cs, 33.10.Jz

\section{Introduction}

The analysis of rotational-vibrational transitions in diatomic molecules is based on the Schrödinger equation

$$
\left[-\frac{\hbar^{2}}{2 m} \frac{\partial^{2}}{\partial q^{2}}+\frac{\hbar^{2} J(J+1)}{2 m q^{2}}+U(q)-E_{v J}\right] \psi_{v J}=0
$$

where $q$ denotes the internuclear separation, $m$ - the reduced mass and $U(q)$ is the internuclear potential energy function. The above equation can be solved analytically for the harmonic [1, 2] and Morse potential [3] using the parabolic expansion of the rotational term in (1), as well as for the Kratzer-Fues potential $[4,5]$ given in the slightly modified form [6-8] as

$$
U(q)=D_{0}\left(1-\frac{q_{0}}{q}\right)^{2}
$$

\footnotetext{
*This research was supported by the Committee for Scientific Research grant 206639101.
} 
where $q_{0}$ is an equilibrium distance and $D_{0}$ is a constant related to the dissociation energy of a molecule. The potential (2) has been recently intensively studied [6-14], as it gives exact analytical solutions which permit a straightforward evaluation of the integrals necessary for investigation of various rovibrational properties, or can be used in the perturbation treatment of higher-order potential terms. Moreover, the potential energy function (2) provides the possibility to include the centrifugal distortion effect [2] occurring in the systems with a rotational degree of freedom [15], in the most direct and natural manner.

Recently, a simple extension of the Kratzer-Fues approach has been proposed in which the set of external semi-empirical parameters is introduced leading to the expansion of the rotational energy of two-atom systems into a continued fraction [16]. The derived formula has been applied in calculation of the rotational energy of rigid, semi-rigid and van der Waals type molecules, giving a quite satisfactory reproduction of molecular spectra.

The main purpose of this paper is to employ the above method in extension of Kratzer-Fues approach to analytical calculation of rovibrational energy and corresponding wave function of diatomic molecules. The modified eigenvalues will be applied in reproduction of rotational and rovibrational spectra of selected diatomic systems. In contrast to our previous proposition the rotational dependence of vibration energy is taken into account, as well as another set of fitted parameters including equilibrium distance and dissociation constant has been employed.

\section{Me'hod and applications}

As was shown in our previous work, the solutions of the Schrödinger equation (1) for rotation-vibration diatomic systems endowed with internuclear potential (2) can be easily modified [16], assuming that the dissociation constant $D_{0}$ depends on the rotational quantum number $J$, and has the continued fraction form

$$
D(J)=D_{0}+\frac{x D_{0} J(J+1)}{1+\frac{y J(J+1)}{1+\frac{x J(J+1)}{1+\ldots}}},
$$

where $\{x, y, z\}$ is a set of semi-empirical parameters. Having substituted $D_{0} \rightarrow$ $D(J)$ in the original equations, one obtains the modified Kratzer-Fues formulae for the rovibrational energy and the corresponding wave functions of diatomic systems

$$
\begin{aligned}
E_{v J} & =D(J)\left\{1-\frac{4 D(J) B(J)^{-1}}{\left[1+2 v+\left(1+4 D(J) B(J)^{-1}\right)^{1 / 2}\right]^{2}}\right\} \\
& +\frac{B_{0} J(J+1)}{1+\frac{B_{0} D_{0}^{-1} J(J+1)}{1+\frac{x J(J+1)}{1+\frac{y J J J+1}{1+\ldots}}}}, \\
\psi_{v J} & =N_{v J}\left(2 \beta(J)_{v} \rho\right)^{\alpha(J)} \exp \left[-\beta(J)_{v} \rho\right] L_{v}^{\gamma(J)}\left(2 \beta(J)_{v} \rho\right),
\end{aligned}
$$

composed of quantities defined in Ref. [16]. In this work we propose to consider the modified formula (4), without neglect of the first term describing rotational 
dependence of the vibration energy, in reproduction of the energy of rotational transitions $(v=0)$ of ${ }^{12} \mathrm{C}^{16} \mathrm{O}, \mathrm{H}^{35} \mathrm{Cl},{ }^{40} \mathrm{Ar}_{2}, \mathrm{HF}$ molecules, as well as rovibrational spectra of ${ }^{13} \mathrm{C}^{16} \mathrm{O},{ }^{12} \mathrm{C}^{18} \mathrm{O}$ and ${ }^{13} \mathrm{C}^{18} \mathrm{O}$ molecules. In order to realize this purpose the set of parameters $\left\{q_{0}, D_{0}, x, y, z \ldots\right\}$, including equilibrium distance, dissociation constant and the additional semi-empirical parameters occurring in (3), will be evaluated by the fitting procedure and used for the calculation of the energy of rotational transitions.

The best values of the molecular parameters will be determined by the linear least squares routine in which the statistical weights, proportional to the inverse of experimental uncertainties, are taken as equal to one. The fitted parameters are presented with their standard errors in Table I which reports also standard deviation $\sigma$ of the $N$-parametric fit, and molecular constants calculated from

$$
\omega=\sqrt{2 D_{0}\left(m q_{0}^{2}\right)^{-1}}, \quad B=\hbar^{2}\left(2 m q_{0}^{2}\right)^{-1}, \quad D=4 B^{3} \omega^{-2} . \quad(6 \mathrm{a}, \mathrm{b}, \mathrm{c})
$$

In the case of the HF molecule the calculated frequencies are compared with those obtained from the $N$-parametric Dunham expansion $(N=3,4,5)$ :

$$
\begin{aligned}
E_{J}= & B J(J+1)-D J^{2}(J+1)^{2}+H J^{3}(J+1)^{3} \\
& -L J^{4}(J+1)^{4}+M J^{5}(J+1)^{5}-\ldots
\end{aligned}
$$

and the simplified fraction continued formula considered in our previous work [16]:

$$
E_{J}=\frac{B_{0} J(J+1)}{1+\frac{B_{1} J(J+1)}{1+\frac{B_{2} J(J+1)}{1+\cdots}}} .
$$

The results of the calculations are collected in Table II. Moreover, the correlation matrices for HF molecular parameters are presented in Tables III and IV. For ${ }^{13} \mathrm{C}^{16} \mathrm{O},{ }^{12} \mathrm{C}^{18} \mathrm{O}$ and ${ }^{13} \mathrm{C}^{18} \mathrm{O}$ molecules the frequencies are calculated from:

(i) The original Kratzer-Fues two-parametric equation $\left(D(J)=D_{0}\right)$.

(ii) The modified Kratzer-Fues formula (4) containing at the most two molecular and one semi-empirical parameters $\left\{q_{0}, D_{0}, x\right\}$, for all the molecules considered.

(iii) The Dunham expansion [17] $E_{v J}=\sum_{k, l} Y_{k l}(v+1 / 2)^{k} J^{l}(J+1)^{l}$, including the comparable number of fitted parameters.

The parameters $\left\{q_{0}, D_{0}, x\right\}$, and the Dunham parameters $Y_{v J}$ evaluated by the fitting procedure and used for the calculation of the energy of rovibrational transitions $(v=0 \rightarrow 1)$ are collected in Table $\mathrm{V}$, which also includes the $\sigma$ standard deviation of the fit, as well as molecular constants $\{\omega, B, D\}$ calculated from $\left\{q_{0}, D_{0}\right\}$ and the formulae $(6 \mathrm{a}-\mathrm{c})$. The experimental frequencies are taken after Refs. [18-21] in the order corresponding to the sequence of the molecules considered.

\section{Discussion}

The obtained results indicate that the modified Kratzer-Fues formula (4) for rovibrational energy of diatomic systems gives the same quality of reproduction of the energy of rotational transitions of ${ }^{12} \mathrm{C}^{16} \mathrm{O}$ and ${ }^{40} \mathrm{Ar}_{2}$ molecules as the 


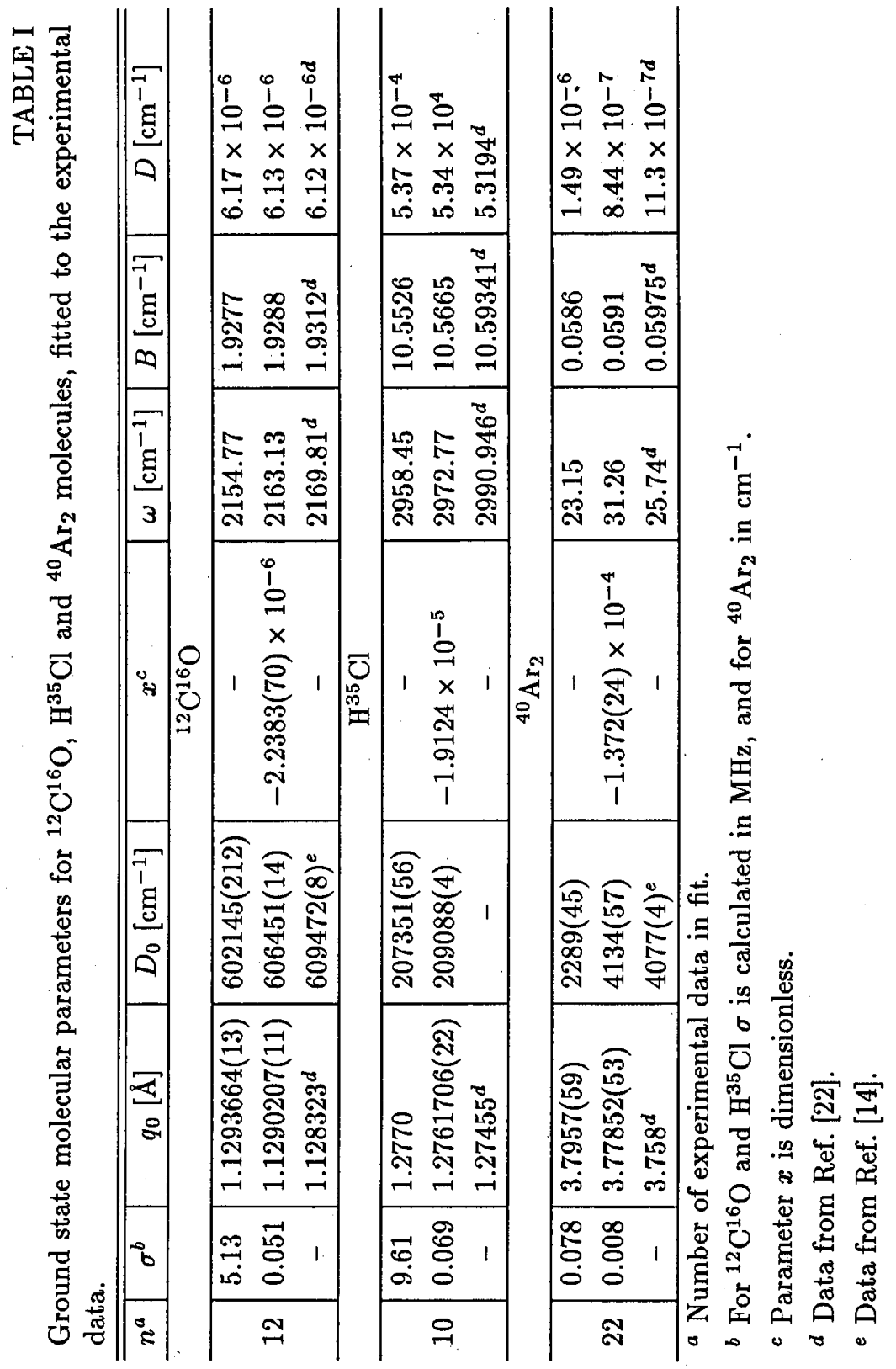


Modified Kratzer-Fues Formula ...

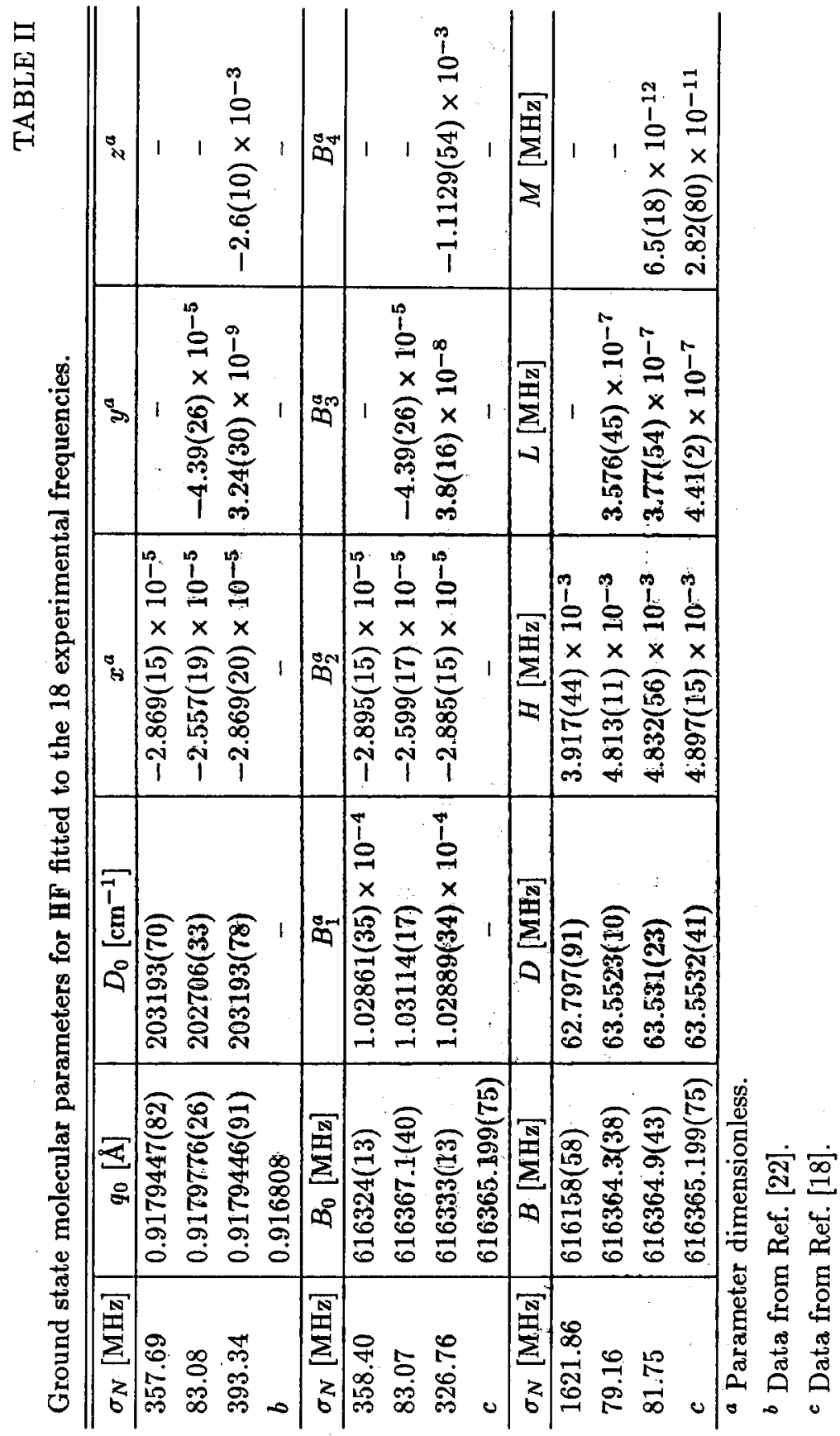


simplified one (8), considered in Ref. [16]. Only in the case of $\mathrm{II}^{35} \mathrm{Cl}$ the accuracy of calculations is slightly improved due to a small correction connected with the rotational dependence of the vibrational term occurring in (4). It may be expected that for highly excited vibrational states the corrections will play an important role, therefore only for low vibrationally excited molecules (as was suggested in our previous work [16]) the simplified continued fraction formula may be applied as an expansion of rotational energy alternative to the Dunham proposal.

Inspection of the data of Table II shows that the rotational spectra of the HF molecule are better reproduced by the Dunham formula (for $N=4,5$ ) than by the continued fraction including the same number of fitted parameters. It is interesting that the application of the fifth continued fraction parameter leads to large deterioration of the accuracy of calculations, therefore only the 4-parametric formula seems to be a physically well-supported equation. However, it is worth noting that the 4-parametric continued fraction, at the comparable standard deviation of the fit obtained using the 5-parametric Dunham expansion, gives a better reproduction of the rotational transitions for high rotational states.

TABLE III

Correlation matrix $N^{a}=4$ for HF molecular parameters.

\begin{tabular}{l|c|c|c|c}
\hline \hline & $q_{0}$ & $D_{0}$ & \multicolumn{1}{c|}{$x$} & \multicolumn{1}{c}{$y$} \\
\hline$q_{0}$ & 1.000 & -0.309 & 0.786 & -0.679 \\
$D_{0}$ & - & 1.000 & -0.964 & 0.899 \\
$x$ & - & - & 1.000 & -0.983 \\
$y$ & - & - & - & 1.000 \\
\hline & $B_{0}$ & $B_{1}$ & $B_{2}$ & \multicolumn{1}{c}{$B_{3}$} \\
\hline$B_{0}$ & 1.000 & 0.905 & 0.757 & -0.651 \\
$B_{1}$ & - & 1.000 & 0.956 & -0.889 \\
$B_{2}$ & - & - & 1.000 & -0.983 \\
$B_{3}$ & - & - & - & 1.000 \\
\hline & $B$ & $D$ & $H$ & $L$ \\
\hline$B$ & 1.000 & -0.914 & 0.786 & -0.679 \\
$D$ & - & 1.000 & -0.949 & 0.899 \\
$H$ & - & - & 1.000 & -0.983 \\
$L$ & - & - & - & 1.000 \\
\hline
\end{tabular}

${ }^{a}$ Number of fitted parameters.

In the case of rovibrational transitions the performed calculations indicate that the modified Kratzer-Fues formula (4), including two molecular and one semi-empirical parameters $\left\{q_{0}, D_{0}, x\right\}$, gives very good reproduction of the observed frequencies and molecular constants for the three isotopic species of $\mathrm{CO}$, 
TABLE IV

Correlation matrix $N^{a}=5$ for $\mathrm{HF}$ molecular parameters.

\begin{tabular}{l|c|c|c|c|c}
\hline \hline & $q_{0}$ & $D_{0}$ & $x$ & $y$ & $z$ \\
\hline$q_{0}$ & 1.000 & 0.851 & -0.755 & -0.159 & 0.167 \\
$D_{0}$ & - & 1.000 & -0.984 & -0.081 & 0.093 \\
$x$ & - & - & 1.000 & 0.064 & -0.076 \\
$y$ & - & - & - & 1.000 & -0.971 \\
$z$ & - & - & - & - & 1.000 \\
\hline & $B_{0}$ & $B_{1}$ & $B_{2}$ & $B_{3}$ & $B_{4}$ \\
\hline$B_{0}$ & 1.000 & 0.944 & 0.865 & 0.310 & 0.191 \\
$B_{1}$ & - & 1.000 & 0.976 & 0.363 & -0.253 \\
$B_{2}$ & - & - & 1.000 & 0.290 & -0.262 \\
$B_{3}$ & - & - & - & 1.000 & -0.505 \\
$B_{4}$ & - & - & - & - & 1.000 \\
\hline & $B$ & $D$ & $H$ & $L$ & $M$ \\
\hline$B$ & 1.000 & -0.752 & 0.567 & -0.477 & 0.425 \\
$D$ & - & 1.000 & -0.965 & 0.924 & -0.893 \\
$H$ & - & - & 1.000 & -0.991 & 0.977 \\
$L$ & - & - & - & 1.000 & -0.996 \\
$M$ & - & - & - & - & 1.000 \\
\hline$a$
\end{tabular}

over a wide range of rotational states. Application of the 3rd parameter $x$ improves the accuracy of calculations, $\sigma$, by about two orders of magnitude relative to the equation based on the original Kratzer-Fues formula, and leads to better results than those obtained from 3-parametric Dunham expansions. Only the 4-parametric Dunham formula reproduces slightly better rovibrational transitions than the modified Kratzer-Fues equation does.

It is worth mentioning here that the application of the 2nd and the following semi-empirical parameters lowers the accuracy of calculations for isotopic species of $\mathrm{CO}$, therefore only the modified formula containing one additional parameter $x$, seems to be a physically well-supported equation.

It is well known that a simple analysis of molecular rovibrational spectra has been realized in the framework of the potential or the Dunham approach. In the potential approach, energy levels are obtained by solving the Schrödinger equation with the interatomic potential expanded in terms of interatomic variables, and the potential coefficients are obtained either by a fit to the experimental energy levels or, if a vailable, by a fit to a theoretically calculated potential. In the above method 


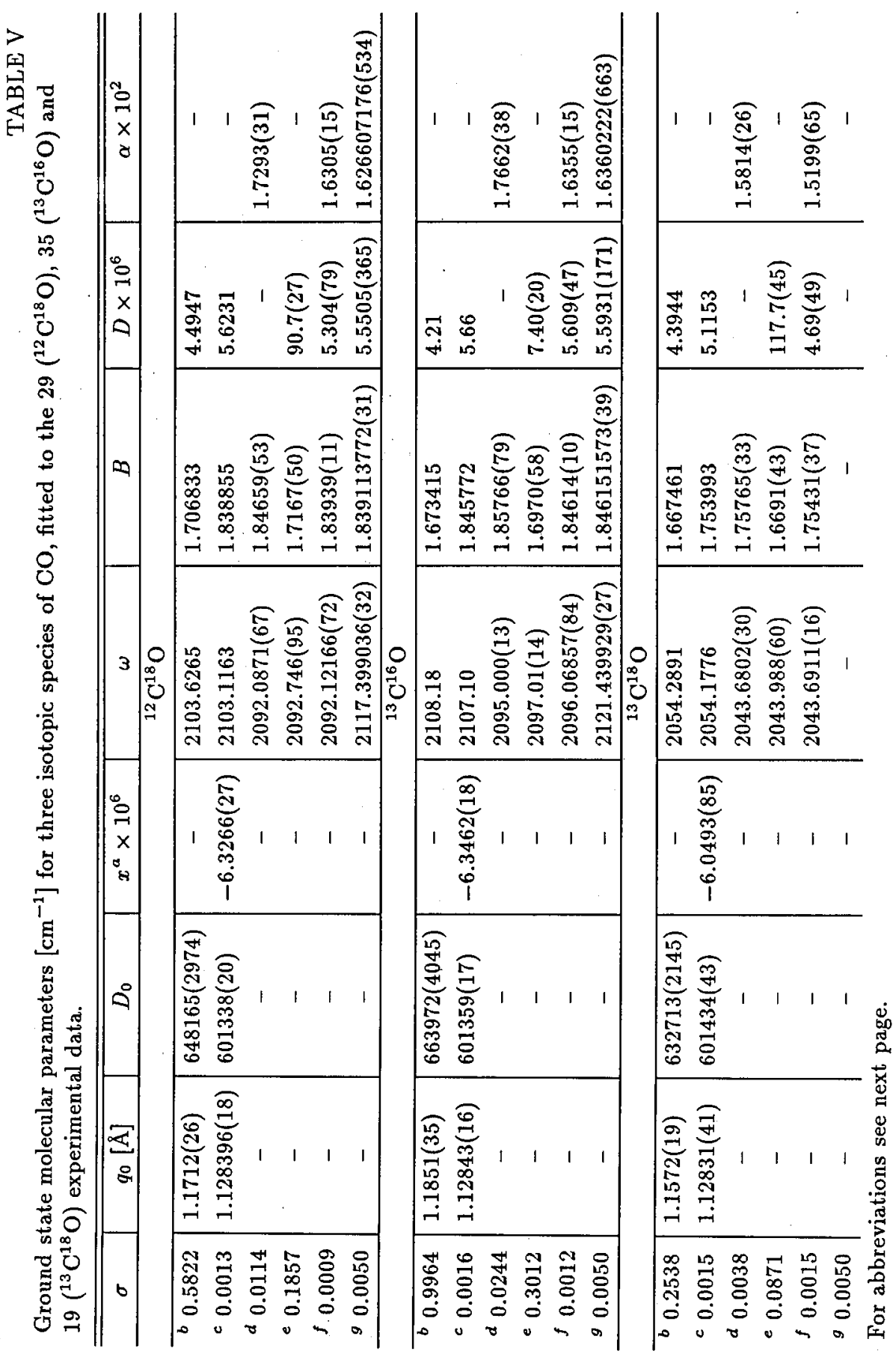


the solution of the Schrödinger equation provides wave functions. In the Dunham approach the rovibrational energy is expanded in terms of vibrational and rotational quantum numbers, and unknown semi-empirical parameters are obtained by a fit to the experimental energy levels. The disadvantage of the Dunham approach is that a power series describing rovibrational energy has a poor convergence, and that Dunham expansion does not provide any information about the wave function of individual states of a molecule. The modification of the analytic Kratzer-Fues formula, by including a set of external semi-empirical parameters, appears to be the third method combining the potential and the Dunham approach, thus permitting the derivation of analytical eigenvalues and eigenfunctions indispensable in a more sophisticated analysis of molecular spectra, and furthermore, allowing the calculation of the matrix elements of quantum-mechanical operators, the Franck-Condon factors and transition intensities [23], which is not possible within the standard Dunham approach.

In our previous work [16], we interpreted the semi-empirical parameter $x$ as an indicator of molecular susceptibility to rotational dissociation. Inspection of Tables I and V reveals that parameter $x$ diminishes with the rigidity of molecules, therefore the above interpretation also holds true for parameters obtained in the presented approach.

Abbreviations for Table V:

a Parameter $x$ is dimensionless.

${ }^{b}$ Parameters calculated from original Kratzer-Fues formula.

c Parameters calculated from modified Kratzer-Fues formula (4).

d Parameters calculated from 3-parametric Dunham expansion $E_{v J}=\omega(v+1 / 2)+[B-\alpha(v+1 / 2)] J(J+1)$.

e Parameters calculated from 3-parametric Dunham expansion $E_{v J}=\omega(v+1 / 2)+[B-D J(J+1)] J(J+1)$.

$f$ Parameters calculated from 4-parametric Dunham expansion $E_{v J}=\omega(v+1 / 2)+[B-\alpha(v+1 / 2)] J(J+1)-D[J(J+1)]^{2}$.

$g$ Data obtained in Ref. [21] on the basis of the 15-parametric Dunham expansion and $163\left({ }^{13} \mathrm{C}^{16} \mathrm{O}\right), 95\left({ }^{12} \mathrm{C}^{18} \mathrm{O}\right)$ and $40\left({ }^{13} \mathrm{C}^{18} \mathrm{O}\right)$ experimental frequencies.

\section{References}

[1] E. Schrödinger, Ann. Phys. 79, 489 (1926).

[2] M. Molski, Acta Phys. Pol. A 81, 485 (1992).

[3] P.M. Morse, Phys. Rev. 34, 57 (1927).

[4] A. Kratzer, Z. Phys. 3, 289 (1920). 
[5] E. Fues, Ann. Phys. (Paris) 80, 367 (1926), 81, 281 (1926).

[6] G. Simons, R.G. Parr, J.M. Finlan, J. Chem. Phys. 59, 3229 (1973).

[7] G. Simons, J.M. Finlan, Phys. Rev. Lett. 33, 131 (1974).

[8] J.M. Finlan, G. Simons, J. Mol. Spetrosc. 57, 1 (1975).

[9] R.W. Bickes, R.B. Bernstein, Chem. Phys. Lett. 26, 457 (1974).

[10] Vl.G. Tyuterev, T.I. Velichko, Chem. Phys. Lett. 104, 596 (1984).

[11] J. Konarski, J. Mol. Spetrosc. 124, 218 (1987).

[12] J. Konarski, Acta Phys. Pol. A74, 235 (1988).

[13] A.V. Burenin, M.Yu. Ryabkin, J. Mol. Spectrosc. 136, 140 (1989).

[14] K. Nakagawa, H. Uehara, Chem. Phys. Lett. 168, 96 (1990).

[15] M. Molski, Acta Phys. Pol. A76, 877 (1989).

[16] M. Molski, J. Konarski, Acta Phys. Pol. A 81, 495 (1992).

[17] J.L. Dunham, Phys. Rev. 41, 713 (1932).

[18] I.G. Nolt, J.V. Radostitz, G. DiLonardo, K.M. Evenson, D.A. Jennings, K.R. Leopold, M.D. Vanek, L.R. Zink, A. Hinz, K.V. Chance, J. Mol. Spetrosc. 125, 274 (1987).

[19] E.A. Colbourn, A.E. Douglas, J. Chem. Phys. 65, 1741 (1976).

[20] D.A. Jennings, K.M. Evenson, L.R. Zink, C. Demuynck, J.L. Destombers, B. Lemoine, J. Mol. Spetrosc. 122, 477 (1987).

[21] D. Chen, K. Narahari Rao, J. Mol. Spetrosc. 61, 71 (1976).

[22] K.P. Huber, G. Herzberg, Constants of Diatomic Molecules, Van Nostrand, New York 1979.

[23] S. Waldenstrøm, K. Razi Naqvi, J. Chem. Phys. 87, 3653 (1987). 